

\title{
Traffic analysis in concurrent multi-channel viewing on P2PTV
}

Koki Mizutani, Takumi Miyoshi, Olivier Fourmaux

\section{To cite this version:}

Koki Mizutani, Takumi Miyoshi, Olivier Fourmaux. Traffic analysis in concurrent multi-channel viewing on P2PTV. Lecture notes in Electrical Engineering, 2015, 339, pp.85-92. 10.1007/978-3-66246578-3_11. hal-01366595

\section{HAL Id: hal-01366595 https://hal.science/hal-01366595}

Submitted on 14 Sep 2016

HAL is a multi-disciplinary open access archive for the deposit and dissemination of scientific research documents, whether they are published or not. The documents may come from teaching and research institutions in France or abroad, or from public or private research centers.
L'archive ouverte pluridisciplinaire HAL, est destinée au dépôt et à la diffusion de documents scientifiques de niveau recherche, publiés ou non, émanant des établissements d'enseignement et de recherche français ou étrangers, des laboratoires publics ou privés. 


\title{
Traffic Analysis in Concurrent Multi-Channel Viewing on P2PTV
}

\author{
Koki Mizutani ${ }^{1}$, Takumi Miyoshi ${ }^{1}$, and Olivier Fourmaux ${ }^{2}$ \\ 1 Shibaura Institute of Technology, Saitama 337-8570, Japan \\ 2 UPMC Sorbonne Universités, Laboratoire d'Informatique de Paris 6, \\ Paris 75005, France
}

\begin{abstract}
In recent years, peer-to-peer (P2P) video streaming services (P2PTV) have attracted much attention because of the ability to decrease the load on the content servers by distributing data delivery function to peers. On the other hand, $\mathrm{P} 2 \mathrm{P}$ overlay networks are oblivious to the physical network topology and thus may cause undesirable traffic straddling on some Internet service providers (ISPs). To optimize P2PTV traffic, several traffic measurements have been studied and revealed the characteristics of P2PTV traffic. However, these studies did not focus on users' behavior and traffic flow of each user. In this paper, we focus on PPTV that is one of the most famous P2PTV services and collect traffic data when multiple channels are viewed at the same time. Through this measurement, we observed the changes of the number of peers, traffic flows, and packet arrival time. As a result, we found the new characteristics of PPTV such as the transmission state of PPTV by monitoring the variation in the number of new arrival peers. Moreover, we could detect video servers by simultaneously analyzing multi-channel PPTV traffic.
\end{abstract}

Keywords: P2PTV, traffic analysis, traffic flow, multi-channel

\section{Introduction}

In recent years, video delivery servers and Internet service providers (ISPs) have been suffering from the increase of traffic load due to the huge demand of multimedia contents. Cisco Systems, Inc. stated that video traffic will be on the increase accounting for 86 percent of all Internet traffic in 2016 [1]. As shown in Fig. 1, peer-to-peer (P2P) video streaming services (P2PTV) have attracted attention as a solution for decreasing the traffic load on content servers due to the nature of $\mathrm{P} 2 \mathrm{P}$ communication that can distribute data delivery function to peers. Currently, P2P streaming applications have become getting popular such as SopCast [2], PPTV [3], and PPStream [4]. In the future, P2P traffic will still increase since video streaming services will shift from the client/server model to the P2P model. To distribute the load on the content servers, P2P communication implements a specific peer selection strategy. In P2PTV communication, a host first selects some peers randomly from the peer list that is offered by the server, and then sends request packets to them. As a result, P2PTV may 
download the video data from farther peers even when there exist some neighbor peers that have the desired video data, and thus establishes inefficient $\mathrm{P} 2 \mathrm{P}$ overlay networks. Moreover, it is difficult for ISPs to deduce P2PTV traffic because of the distribution of peers around the world. Therefore, P2P traffic optimization is one of the biggest problems for ISPs.

To optimize P2PTV traffic, several traffic measurements have been studied and revealed its characteristics [5]-[7]. However, these studies are not enough to reveal users' behavior since they only inspected a steady-state traffic in a long duration of time on the same channel. To study P2PTV traffic sufficiently, we need to inspect the behavior of peers and to compare the characteristics of P2PTV among multiple channels. This paper focuses on analyzing traffic characteristics and users' behavior among multiple channels on PPTV, which is one of popular P2PTV applications. We run PPTV applications on multiple PCs to view multiple channels and collect traffic data at the same time. As a result, we show the following characteristics: (1) frequencies in the use of the same peers among multiple channels, (2) the transmission state of PPTV by comparison between the variation in the number of new arrival peers and long duration flows, and (3) the possibility to find super peers that provide stable video streaming by analyzing traffic flows among multiple channels.

\section{Related Work}

Several conventional studies have investigated the characteristics of P2PTV applications including PPStream, PPTV, SopCast, etc. Hei et al. analyzed the steady state of P2PTV traffic on PPLive (former name of PPTV) with a crawler that can automatically send and receive control packets [5]. They discovered the variation in the characteristics of throughput, users' behavior, and the number of connections on PPLive. They also concluded that users tend to change their channels to others on the desired channel selection. This behavior is similar to


Fig. 1. Network model of video streaming services. 
the channel zapping on analog TV. Jia et al. analyzed the characteristics of the traffic on PPStream with crawler [6]. They clarified the geographical characteristics, the variation in the number of peers, and the ratio of sent and received packets. They concluded that PPStream has a hierarchical mesh network topology including super nodes eager to send the video data to many users. Silverston et al. analyzed P2PTV traffic on PPStream and SopCast measuring on multiple points in different locations of the world [7]. They clarified the number of AS hops and the ratio of download and upload data from the viewpoint of measurement locations. They concluded that the P2PTV applications have not been equipped with any traffic localization mechanism because many peers received video data from not own countries but others. In addition, they indicated that P2PTV also has no fairness in sharing the video data because of the differential ratio of download and upload data among peers.

The above studies analyzed P2PTV traffic in a long duration of time and revealed the characteristics of P2PTV traffic, the geographical location of peers, the overlay topology, etc. However, they are not enough to study the characteristic of P2PTV since they did not focus on the characteristics of users' behavior such as traffic per each user. In addition, these studies were measured only on single channel. To reveal and generalize the characteristic of P2PTV traffic, it is essential to investigate P2PTV traffic on multiple channels.

\section{Objective of P2PTV Traffic Measurement}

These days, various analyses of P2PTV traffic have been performed. However, these studies are not enough to reveal the P2PTV traffic thoroughly considering users' behavior and the extraction of P2PTV characteristics among multiple channels.

In this paper, we present a novel measurement experiment for multi-channel viewing by using multiple measurement PCs. We collect traffic data among multiple channels on P2PTV and analyze the traffic per each user. Based on the collected traffic, we firstly determine single-channel peers and multi-channel peers among peers that the P2PTV application connects to. A single-channel peer is defined as the peer that appears only in one channel, i.e., the peer is observed on a single channel. A multi-channel peer is defined as the peer that appears in two or more channels, i.e., the peer is observed on multiple channels. On $\mathrm{P} 2 \mathrm{PTV}$, it is not sure that measurement PCs can observe the same peers among multiple channels due to the nature of random peer selection. Therefore, we examine frequencies in the use of the same peers and define peers observed on various channels as multi-channel peers. In our second analysis, we examine the transmission state of P2PTV on a specific channel. This can be done by analyzing the variation in the number of new arrival peers and long duration flows. In particular, if many new arrival peers are intensively found on a channel, we can assume that the measurement PC is trying to search some stable peers at that time. Finally, we specify which peers could be a video server by analyzing traffic from multi-channel peers on each measurement PC. If some measurement 
PCs can simultaneously observe peers with the same IP address among multiple channels, we can decide that these peers are video servers deployed for providing stable P2PTV services.

\section{Experimental Setting}

This section describes our experimental environment. We focused on PPTV that is one of the most famous P2PTV services. We measured traffic for twelve hours (from 12:00 JST to 23:59) on 18th December 2013 on PPTV using ten measurement PCs including six virtual machines. As shown in table 1, we prepared three types of measurement PCs including high-, medium-, and low-performance PCs assuming actual users' environment. Every PC has an Internet connection provided by FLET'S HIKARI NEXT, 100 Mbps optical access service on next generation network (NGN) via Plala HIKARI Mate as an ISP in Japan. For capturing and monitoring traffic, Wireshark [8], a well-known packet sniffer, is installed on every measurement PC. As shown in Fig. 2, we viewed different live streaming contents through 10 popular channels based on the official popularity ranking on PPTV.

In the measurement PCs, we extracted only packets with not less than 1000 Bytes as video packets to cut off control packets and then analyzed only video traffic flows. Note that a flow represents a group of packets consecutively received from the same IP address. If the interval between two consecutive packets from the same IP address is more than 60 seconds, however, we regard them as different flows. Moreover, some flows may continue to exist even after our measurement. Therefore, we excluded some flows that maintained connections within the last 60 seconds of the measurement.

Table 1. Performance of measurement PCs.

\begin{tabular}{c|c|c|c|c}
\hline Performance & CPU & \# of cores & Memory (GB) & OS \\
\hline High & $\begin{array}{c}\text { Intel Core } \\
\text { i5-3470S 2.90GHz }\end{array}$ & 4 & 12 & Windows 7 \\
\hline Medium & $\begin{array}{c}\text { Intel Core } \\
\text { i3-3217U 1.80GHz }\end{array}$ & 2 & 8 & Windows 7 \\
\hline Low & $\begin{array}{c}\text { Intel Core } \\
\text { i5-3470S 2.90GHz }\end{array}$ & 1 & 2 & Windows 7 \\
\hline
\end{tabular}



Fig. 2. Experimental environment. 


\section{Analysis of P2PTV Traffic}

In this section, we analyze PPTV traffic measured in our experiment.

\subsection{Ratio of multi-channel peers}

First, we extracted multi-channel peers that appeared in multiple channels. Then, we calculated the ratio of multi-channel peers to the total number of peers appearing in each channel. As shown in table 2 , for instance on channel 1 , a measurement PC observed 94 multi-channel peers and 793 peers in total, and therefore the ratio of multi-channel peers accounted for 11.9 percent. Moreover, we categorized channels considering the total number of peers into two groups of channels; popular channel group that has 1000 or more peers and unpopular channel group that has less than 1000 peers. In this experiment, channels $1,3,4$, 5,6 , and 10 are categorized into unpopular channel group while channels $2,7,8$, and 9 are categorized into popular channel group. The number of multi-channel peers accounted for 5 to 12 percent of the total number of peers on each channel.

\subsection{Analysis of traffic flows}

We analyzed traffic flows on channel 1 and 9, which represent the unpopular and popular channel groups, respectively. From Figs. 3 to 6 show the variation in peer numbers, the order of peers arrival, for 45000 seconds. In this experiment, we extracted long duration flows that connected to the same peers for not less than 1200 seconds (20 minutes). Firstly, Figs. 3 and 5 show all traffic flows on channel 1 and 9 , respectively. In addition, Figs. 4 and 6 pick up long duration flows from Figs. 3 and 5, respectively. In each of figures, orange lines show traffic flows of single-channel peers, and blue lines show those of multi-channel peers. At the beginning of each measurement, as shown in Figs. 3 and 5, measurement PCs drastically connected to multi-channel peers. Moreover, we found a relationship between the number of new arrival peers and long duration flows. In particular, the comparison between Figs. 3 and 4 indicates that a huge increase of new

Table 2. Statistics of peers and multi-channel peers.

\begin{tabular}{r|r|r|r}
\hline CH\# & Total peers & Multi-channel peers & Ratio of multi-channel peers (\%) \\
\hline 1 & 793 & 94 & 11.9 \\
\hline 3 & 838 & 66 & 7.9 \\
\hline 4 & 680 & 70 & 10.3 \\
\hline 5 & 514 & 56 & 10.9 \\
\hline 6 & 665 & 62 & 9.3 \\
\hline 10 & 544 & 64 & 11.8 \\
\hline 2 & 1882 & 149 & 7.9 \\
\hline 7 & 1810 & 94 & 5.2 \\
\hline 8 & 1516 & 88 & 5.8 \\
\hline 9 & 1440 & 119 & 8.3 \\
\hline
\end{tabular}


arrival peers is observed on the disappearance of long duration flows from 23000 to 25000 seconds.

To clear the above relationship, we focused on the variation in the number of new arrival peers and long duration flows for 45000 seconds on channel 1 and 9 , shown in Figs. 7 and 8, respectively. In each of figures, red lines show the number of new arrival peers, and blue lines show the number of long duration flows. In Fig. 7, a huge increase of new arrival peers is observed while long duration flows disappear from 23000 to 25000 seconds. We conclude that the variation in the number of new arrival peers is a very important point to understand the transmission state of PPTV. We assume that PPTV lost stable peers to download the video data due to the immense decrease of long duration flows and then started searching new stable peers. According to this assumption, we can deduce the transmission state of PPTV by monitoring the variation in the number of new arrival peers. We found the similar tendency in Fig. 8 regardless of the number of peers in channels.



Fig. 3. All traffic flows on channel 1.



Fig. 5. All traffic flows on channel 9.



Fig. 4. Long duration flows on channel 1.



Fig. 6. Long duration flows on channel 9. 


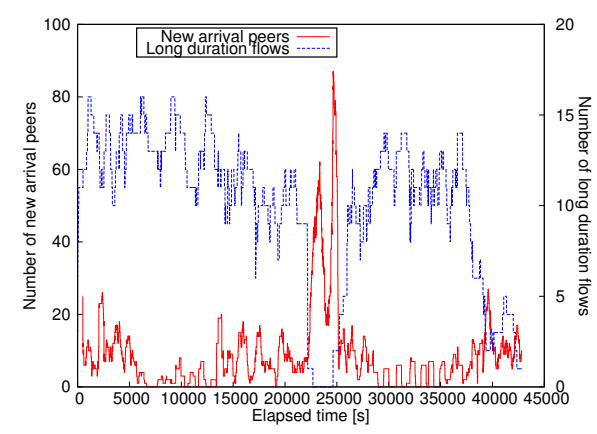

Fig. 7. Relationship between the changes of new arrival peers and long duration flows on channel 1.



(a) From Super peer

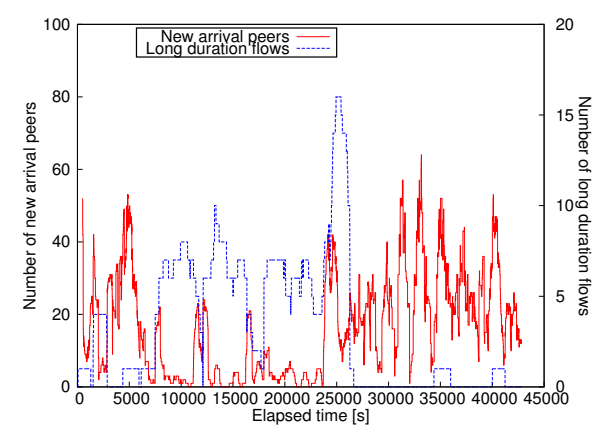

Fig. 8. Relationship between the changes of new arrival peers and long duration flows channel 9.



(b) From Multi-channel peer

Fig. 9. Flows from specific peers appearing on each measurement PC.

\subsection{Behavior of multi-channel peers}

Finally, we try to find the super peer among multiple channel. Fig. 9 shows the flows from a multi-channel peer on each channel viewed by each measurement PC. In Fig. 9 (a), measurement PCs simultaneously observed the same peer among multiple channels for a long duration. Thus, we assume that this peer was a video server called super peer, which provides stable video streaming services dedicating itself to sending video packets. On the contrary, in Fig. 9 (b), measurement PCs observed a channel transition of another multi-channel peer. This multi-channel peer was not observed simultaneously by several measurement PCs and disappeared shortly. We assume that this kind of behavior is users because user peers do not have intension to provide stable video streaming services and just select their preferred channels by themselves. According to these 
analyses, we can simply detect video servers with video traffic measurements on multiple channels.

\section{Conclusion}

In this paper, we discovered new characteristics of PPTV traffic with measurement experiment among multiple channels. Firstly, we calculated the ratio of multi-channel peers to the total number of peers appearing in each channel and found that multi-channel peers accounted for 5 to 12 percent of the total number of peers observed on each channel. Secondly, we found that huge increases of new arrival peers were observed on the disappearance of long duration flows. According to this characteristic, we can deduce the transmission state of PPTV by monitoring the variation in the number of new arrival peers. Finally, we can also detect super peers by monitoring the same peer observed among multiple channels simultaneously.

For the future works, we are going to analyze traffic characteristics on other P2PTV applications with measurement experiment among multiple channels simultaneously. Moreover, we will configure a traffic optimization system that controls P2PTV traffic considering the transmission state of P2PTV.

\section{Acknowledgment}

This study was partly supported by JSPS KAKENHI Grant Number 26420370.

\section{References}

1. Cisco Systems, Inc., "Cisco visual networking index: forecast and methodology, 2011-2016," White paper, May 2012.

2. SopCast. http://www.sopcast.org/

3. PPTV. http://www.pptv.com/

4. PPStream. http://www.pps.tv/

5. X. Hei, C. Liang, J. Liang, Y. Liu, and K.W. Ross, "A measurement study of a largescale p2p iptv system," IEEE Trans. on Multimedia, Vol. 9, Issue 8, pp. 1672-1687, Dec. 2007.

6. J. Jia, C. Li, and C. Chen, "Characterizing ppstream across internet," 2007 IFIP Int'l Conf. Netw. and Parallel Comput. (NPC'07) Workshops, pp. 413-418, Sept. 2007.

7. T. Silverston, O. Fourmaux, K. Salamatian, and K. Cho, "On fairness and locality in p2p-tv through large-scale measurement experiment," IEEE Global Telecommun. Conf. (GLOBECOM 2010), Dec. 2010.

8. Wireshark. http://www.wireshark.org/ 